\title{
Endemic Jeffrey Pine Beetle Associates: Beetle/Mite Fungal Dissemination Strategies and Interactions That May Influence Beetle Population Levels
}

\author{
Javier E. Mercado ${ }^{1, *}$, Beatriz Ortiz-Santana ${ }^{2}$ and Shannon L. Kay ${ }^{1}$ \\ 1 USDA/FS/Rocky Mountain Res. Sta., Fort Collins, CO 80526, USA; shannon.kay@usda.gov \\ 2 USDA/FS/Northern Res. Sta., Madison, WI 53726, USA; beatriz.ortiz-santana@usda.gov \\ * Correspondence: javier.mercado@usda.gov; Tel.: +1-(970)-498-1387
}

check for

updates

Citation: Mercado, J.E.;

Ortiz-Santana, B.; Kay, S.L. Endemic

Jeffrey Pine Beetle Associates:

Beetle/Mite Fungal Dissemination

Strategies and Interactions That May Influence Beetle Population Levels.

Microorganisms 2021, 9, 1641.

https://doi.org/10.3390/

microorganisms 9081641

Academic Editor: Elisa Gamalero

Received: 16 June 2021

Accepted: 29 July 2021

Published: 31 July 2021

Publisher's Note: MDPI stays neutral with regard to jurisdictional claims in published maps and institutional affiliations.

Copyright: (C) 2021 by the authors. Licensee MDPI, Basel, Switzerland. This article is an open access article distributed under the terms and conditions of the Creative Commons Attribution (CC BY) license (https:/ / creativecommons.org/licenses/by/ $4.0 /)$.

\begin{abstract}
Fungal and mite associates may drive changes in bark beetle populations, and mechanisms constraining beetle irruptions may be hidden in endemic populations. We characterized common fungi of endemic-level Jeffrey pine beetle (JPB) in western USA and analyzed their dissemination by JPB (maxillae and fecal pellet) and fungivorous mites to identify if endogenous regulation drove the population. We hypothesized that: (1) as in near-endemic mountain pine beetle populations, JPB's mutualistic fungus would either be less abundant in endemic than in non-endemic populations or that another fungus may be more prevalent; (2) JPB primarily transports its mutualistic fungus, while its fungivorous mites primarily transport another fungus, and (3) based on the prevalence of yeasts in bark beetle symbioses, that a mutualistic interaction with blue-stain fungi present in that system may exist. Grosmannia clavigera was the most frequent JPB symbiont; however, the new here reported antagonist, Ophiostoma minus, was second in frequency. As hypothesized, JPB mostly carried its mutualist fungus while another fungus (i.e., antagonistic) was mainly carried by mites, but no fungal transport was obligate. Furthermore, we found a novel mutualistic interaction between the yeast Kuraishia molischiana and G. clavigera which fostered a growth advantage at temperatures associated with beetle colonization.
\end{abstract}

Keywords: mutualist; antagonist; blue-stain fungus; fungal biome; population regulation

\section{Introduction}

Phytopathogenic fungi, such as several blue-staining species associated with bark beetles, establish a variety of symbiotic interactions with beetles that range from obligate mutualisms to antagonisms. Most of our understanding of these interactions is derived from a few bark beetle species that undergo populations irruptions. A well-studied example in western North America is that of the blue-stain fungus Grosmannia clavigera (Rob.-Jeffr. \& R.W. Davidson) Zipfel, Z.W. de Beer \& M.J. Wingf. This fungus gains transport to suitable host trees aided by the mountain pine beetle (Dendroctonus ponderosae, Hopk.), hereafter MPB. In return, G. clavigera provides nutrition to the beetle's larvae developing in the phloem, making their relationship mutually beneficial (mutualistic) to both organisms. In addition to nutrition, bark beetles derive other benefits from their fungal associates such as the detoxification of harmful oleoresin compounds [1]. Additionally, bark beetle chemical communication is partly mediated by yeasts, such as Kuraishia capsulata (Wick.) Y. Yamada, K. Maeda \& Mikata and Ogataea pini (Holst) Y. Yamada, M. Matsuda, K. Maeda \& Mikata, that synthesize and release verbenone used by searching beetles to recognize host saturation levels during beetle attack [2]. Given how fungi can influence their associated bark beetles, a good understanding of their prevalence during contrasting beetle population levels (i.e., collapsing and endemic vs. irruptive) can help elucidate their effect on shifting population levels; however, studies on collapsing or endemic-level bark beetle populations are rarely performed. Studies looking at symbiotic fungal communities of Dendroctonus 
bark beetles have found that their frequency can change over time with trends directly associated with fluctuations of their carrier beetle populations, strongly suggesting the type of interaction occurring. For example, an increase of Ophiostoma minus in the southern pine beetle (Dendroctonus frontalis Zimmermann) can drive a decline of the beetle's population, representing an antagonistic interaction that regulates their population [3,4]. Conversely, in the MPB a decrease in the frequency of nutritional blue-stain fungi, which is abundant during epidemic-population levels, trended directly with a decline in the insect's population size, signaling a mutualistic interaction between the two organisms [5]. Therefore, monitoring the trends of important fungal associates in irruptive bark beetles during population shifts could help explain and perhaps predict fluctuations of bark beetle populations.

The ways in which blue-stain fungi disseminate their spores between carrying beetles and host trees are varied, and identifying if evolutionary adaptations of insects, phoretic mites, or the fungi influence their frequency in these systems could aid in determining the type of symbiotic interaction being established. Fungi associated with Dendroctonus bark beetles can be carried internally or externally on beetles, or by their phoretic mites [6,7]. Yeasts can be carried in the beetle's digestive tract, especially in the gut [8,9], yet other forms of dissemination may exist. For instance, we know that fungi can survive enzymatic action in the gut of MPB [10], and although their survival of digestive elimination has been suggested [11], this is not fully understood.

One closely related species to the MPB is the Jeffrey pine beetle (Dendroctonus jeffreyi, Hopk.), hereafter JPB. The two species resemble each other, both morphologically and genetically [12-15]. These two species also share a unique trait within the genus Dendroctonus, the possession of a pit or mycangium (specialized fungus harboring site) contained within each maxilla (maxillae in text) for the dissemination of fungi [16]. Besides the above morphological similarities, these two bark beetles also rely on the common nutritionally beneficial fungus $G$. clavigera, which complements the nutrients they obtain from the phloem. Although MPB also obtains nutritional benefits from Leptographium longiclavatum S.W. Lee, J.J. Kim \& C. Breuil and Ophiostoma montium (Rumbold) von Arx, its phoretic mites also carry them, making its mutualism with G. clavigera one facultative. Grosmannia clavigera is the only known nutritional blue-stain fungus of JPB and we do not know if its phoretic mites carry it. This makes the type of mutualism between JPB and G. clavigera one obligate. Additionally, both species can carry a variety of other fungi and yeasts that have unresolved effects on their populations.

As in several other bark beetle species, JPB fungal associates have been studied during moderate population irruptions, when access to adequate samples is best. Nine fungi are associated with JPB (Table 1), of which the blue-staining G. clavigera is commonly carried by the beetle across most of its range [17]. But the other two MPB blue-stain fungal mutualist, O. montium and L. longiclavatum, were reported each once before from JPB in California [18,19]. The long, clavate, and septate conidia morphologically distinguish them from most related taxa, but separating them often requires molecular identification. Therefore, as in the MPB, L. longiclavatum may be more prevalent in JPB populations than expected [5]. Most yeasts transported by JPB belong to the Order Saccharomycetales, of which K. capsulata and Nakazawaea holstii (Wick.) Y. Yamada, K. Maeda \& Mikata are the most prevalent [20]. Although the function of many yeasts in bark beetle symbiotic systems is not completely understood, their ubiquity suggests that many of these are at least commensals, benefiting by gaining transport to new hosts with the beetles. In culture and trees, yeasts often grow together with blue-stain fungi which makes separating them during isolations difficult [21], but their tolerance of each other suggests that a non-antagonistic symbiotic relationship may exist between them. 
Table 1. Previously documented fungal associates of Jeffrey pine beetle and the structure from which they have been collected.

\begin{tabular}{lccc}
\hline \multicolumn{1}{c}{ Species } & BeetleStructure & Locality & Reference \\
\hline Ophiostoma ips/montium & unreported & Stanislaus NF, CA & {$[18]$} \\
Grosmannia clavigera & Maxillae & S. Bernandino Mnts., CA and NV & {$[17]$} \\
Leptographium longiclavatum & Maxillae & Monitor Pass, Toiyabe NF, CA & {$[19]$} \\
Candida silvicola & Gut & Northern California & {$[20]$} \\
Diutina rugosa & Gut & Northern California & {$[20]$} \\
Kuraishia capsulata & Gut & Northern California & {$[20]$} \\
Nakazawaea holstii & Gut & Northern California & {$[20]$} \\
Priceomyces haplophilus & Gut & Northern California & {$[20]$} \\
Spencerozyma crocea & Gut & Northern California & {$[20]$} \\
\hline
\end{tabular}

In this study, we examined the composition and frequency of associated fungi in an endemic JPB population sample. We isolated fungi and yeasts from JPB maxillae, rectal fecal pellets, and mites. We aimed to identify relationships between the frequency of associated fungi to the beetle's endemic-population level, using their dissemination method and known function in other bark beetle systems to derive a better understanding of their role in the JPB system. Based on previous work and the endemic-population level of our sample, we hypothesized that: (1) as a population regulation factor noted in the MPB, G. clavigera would either be less abundant than in non-endemic populations or that another fungus may be more prevalent; (2) as in other Dendroctonus species, JPB would transport primarily mutualistic fungi, while the associated fungivorous mites would transport primarily nonmutualistic fungi to JPB, and (3) based on the prevalence of yeasts in bark beetle symbioses, that a mutualistic interaction with blue-stain fungi commonly present in the system may exist. Our findings would not only increase our knowledge about JPB associates but would reinforce the hypothesis suggesting that an endogenous population control of irruptive bark beetle populations is exerted by their microbial associates.

\section{Materials and Methods}

\subsection{Bark Beetle Sample Collection}

Jeffrey pine beetles were collected in the Lassen N.F. on 23 June 2017. Jeffrey pine beetle populations fluctuate between endemic and moderately irruptive levels. In the general area around the collection site, recent JPB populations were moderately irruptive from 1991 to 1997, 2001 to 2004, and 2014 to 2016. At the time of collection, the species activity was experiencing a declining trend relative to their climax in 2016 [22]. Specific to our sample, the declining population was considered to be endemic, given the difficulty in finding trees affected by the beetle and its attack behavior. Fifty-seven beetles were individually collected from beneath the bark of a 300-year-old Jeffrey pine before their early summer dispersal flight period. Beetles were immediately stored in a cooler and shipped refrigerated to the Rocky Mountain Research Station in Fort Collins. Due to their similarity with the MPB and their overlapping range, we carefully identified JPB beetles using available morphological identification keys [12]. We compared the genitalia (male endophallus) to that of D. ponderosae and found it to be different. We illustrate JPB's endophallus here for the first time (Figure 1).

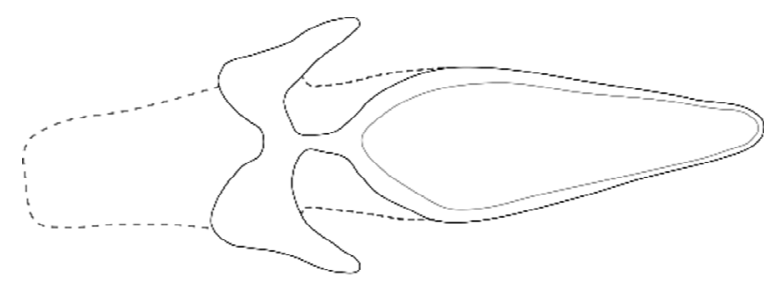

Figure 1. We used the undescribed endophallus of Jeffrey pine beetle (illustrated) as new character to distinguish this species from the similar mountain pine beetle and found it to be a useful character. 


\subsection{Isolation of Fungi from Beetle Structures and Mites}

We thawed beetles for four minutes before dissections to remove their maxillary cardines, fecal pellets, and fungivorous mites, if present. We cultured the pellets and mites directly into the agar plates without performing a rinse, whereas two sequential rinses on a drop of molecular grade sterilized water were performed on exterior surfaces of the maxillary cardines (maxillae hereafter) before culturing. We used malt extract agar $(1.5 \%$ MEA, Bacto Malt Extract, BD Biosciences, San Jose, CA, USA) for the direct culture of the three targets to obtain prevailing JPB fungal associates. We used a direct culture method instead of sequential dilutions and streaking method since competition between common fungal associates of related Dendroctonus beetles is non-significant [23]. We examined cultures daily for 21 days to recover any distinct fungal colonies.

\subsection{Single Fungal Strain Isolations}

In Dendroctonus bark beetle's fungal isolates, yeasts are typically the first associates to grow. These usually surround other fungal growth making their separation difficult. We tried two methods to recover fungi from yeast overgrowth: single spore isolation and hyphal tip harvesting, of which the latter was used for this study. Material for single spore isolation came from two sources, direct spore transferences from aerial conidiophores growing on MEA and sterilized phloem sections $(2 \times 4 \mathrm{~cm})$ placed inside sterile and parafilm sealed $60 \mathrm{~mm}$ petri dishes. We harvested hyphal tips from above as well as from below the agar. This last strategy was the most efficient in reducing yeast contamination when harvesting hyphal tips.

\subsection{Morphological Determination}

We studied cultural and microscopic characters of pure strains and made identifications based on authoritative sources [24,25]. Strains' cultural and morphological characters used in diagnostics were color, growth characteristics of the margin, and patterns of spore production in MEA plates. In addition, from conidia produced in the sterilized phloem sections, the microscopic diagnostic characters used were conidiophore type and length, conidial shape, and ratio. We identified fungivorous mites only to the Family taxonomical level. All isolates were deposited in the Center for Forest Mycology Research Culture Collection (CFMR) in Madison, WI, USA.

\subsection{Molecular Data}

In the present study, we generated DNA sequences of the nuclear ribosomal ITS2LSU (partial $5.8+$ ITS2 + partial 28S) and the protein-coding gene $\beta$-tubulin (partial gene) from 21 cultures. We performed DNA extraction, amplification, and sequencing as indicated in Mercado and others [5]. DNA sequences were used for molecular identification and phylogenetic analyses. A total of 20 ITS2-LSU and $\beta$-tubulin sequences were newly generated while 35 ITS2-LSU and $44 \beta$-tubulin were retrieved from GenBank. The selection of sequences from GenBank [26] was performed using BLASTn search results and species previously grouped within the Grosmannia and Ophiostoma clades [27-31]. The information for these isolates is provided in Table 2. Newly generated sequences were edited with Sequencher 4.8 (Gene Codes Corp., Ann Arbor, MI, USA) and were deposited in GenBank (MZ297376-MZ297395, MZ296739-MZ296755).

\subsection{Sequences Alignment and Phylogenetic Analyses}

To align the DNA sequences and to construct our phylogenetic trees we proceeded as follows. Bayesian inference (BI) and Maximum likelihood (ML) phylogenetic analyses were executed on two datasets: Ophiostoma ITS2-LSU $+\beta$-tubulin and Grosmannia ITS2-LSU $+\beta$-tubulin. DNA sequences were aligned using Clustal W2.1 through CIPRES Science Gateway [32] and were manually adjusted with AliView 1.18 [33]. Final alignments were deposited in TreeBASE (TB2:S28351, S28352). The loci were concatenated using Sea View 4 [34] and the two-locus datasets were appended with a MrBayes block, partitioned the 
data by gene, codon position, and noncoding region for BI analysis. The nexus files were converted into phylip files and run through jModelTest 2 [35] in CIPRES to estimate the best substitution model for the analyses. The best-fit model estimated for the Ophiostoma ITS2$\mathrm{LSU}+$ Btubulin dataset was $\mathrm{TrN}+\mathrm{G}$ (corresponding to SYM in MrBayes with parameters nst $=6$ and rates $=$ gamma), whereas for Grosmannia ITS2-LSU + Btubulin dataset was $\operatorname{TrN}$ (corresponding to SYM in MrBayes with parameters nst $=6$ and rates $=$ equal). Bayesian inference analysis was performed using MrBayes 3.2.2 [36] on XSEDE through CIPRES. The parameters mentioned above were used for each dataset for 1,000,000 generations in two runs and four chains with trees sampled every 100 generations. The burn-in period was set to 0.25 . Maximum likelihood analyses were performed using RAxMLHPC2 on XSEDE through CIPRES under the GTR model with gamma distributed rate heterogeneity and 1000 rapid bootstrap replicates; other parameters were kept at their default settings. Phylogenetic trees were visualized and edited in FigTree 1.4.4 [37] and rooted to midpoint. Final trees were edited in Adobe Illustrator CC 2018 (San José, CA, USA). Strong support values of clades are $>90 \%$ in $\mathrm{ML}$ and $>0.95$ posterior probabilities (PPs) in BI analyses, whereas moderate support values are $>70 \%$ and $>0.90$, respectively. The bootstrap frequencies $(>50 \%)$ and posterior probabilities $(>0.80)$ are shown on branches.

Table 2. Taxa, isolate information, location, and GenBank accession numbers of the samples used in the phylogenetic analyses. New sequences appear in bold.

\begin{tabular}{|c|c|c|c|c|}
\hline Species & Isolate & Location & ITS-LSU & $\beta$-tubulin \\
\hline \multirow[t]{2}{*}{ Grosmannia aurea } & UC03DL06 & Canada-AB & GU370267 & GU370181 \\
\hline & UC16G21 & Canada-AB & GU370293 & GU370207 \\
\hline \multirow[t]{16}{*}{ G. clavigera } & CA-JM-71 & USA-CA & MZ297376 & MZ296739 \\
\hline & CA-JM-72 & USA-CA & MZ297377 & MZ296740 \\
\hline & CA-JM-73 & USA-CA & MZ297378 & MZ296741 \\
\hline & CA-JM-74 & USA-CA & MZ297379 & MZ296742 \\
\hline & CA-JM-75 & USA-CA & MZ297380 & MZ296743 \\
\hline & CA-JM-76 & USA-CA & MZ297381 & MZ296744 \\
\hline & CA-JM-77 & USA-CA & MZ297382 & MZ296745 \\
\hline & CA-JM-78 & USA-CA & MZ297383 & MZ296746 \\
\hline & CA-JM-79 & USA-CA & MZ297384 & MZ296747 \\
\hline & CA-JM-80 & USA-CA & MZ297385 & MZ296748 \\
\hline & CO-JM-17 & USA-CO & KY940832 & KY940842 \\
\hline & CO-JM-41 & USA-CO & KY940833 & KY940843 \\
\hline & SL-Kw1407 & Canada-BC & AY544615 & AY263195 \\
\hline & UC14G23 & Canada-AB & GU370266 & GU370180 \\
\hline & UC35DL49 & Canada-AB & GU370288 & GU370202 \\
\hline & UM01G12 & Canada-AB & GU370274 & GU370188 \\
\hline \multirow[t]{2}{*}{ G. huntii } & CBS398.77 & Canada & AY707208 & AY707194 \\
\hline & UAMH4997 & Canada & AY544617 & AY349023 \\
\hline \multirow[t]{2}{*}{ G. robusta } & CMW 668 & USA-ID & AY553397 & AY534945 \\
\hline & CMW 2805 & USA-ID & AY553396 & AY534944 \\
\hline \multirow[t]{6}{*}{ Leptographium longiclavatum } & CO-JM-8 & USA-CO & KY940824 & KY940834 \\
\hline & CO-JM-42 & USA-CO & KY940826 & KY940836 \\
\hline & CO-JM-46 & USA-CO & KY940830 & KY940840 \\
\hline & SL-Kp11 & Canada-BC & AY816687 & AY816712 \\
\hline & SL-Kw1436 & Canada-BC & AY816686 & AY288934 \\
\hline & UL13G22 & Canada-BC & GU370300 & GU370214 \\
\hline \multirow[t]{2}{*}{ L. terebrantis } & UC38DL41 & Canada-AB & GU370295 & GU370209 \\
\hline & UC48G19 & Canada-AB & GU370272 & GU370186 \\
\hline \multirow[t]{2}{*}{ L. wingfieldii } & CMW 2095 & France & AY553400 & AY534948 \\
\hline & CMW 2096 & France & AY553398 & AY534946 \\
\hline \multirow[t]{2}{*}{ Ophiostoma bicolor } & 3YT4P-Ob & Canada & DQ268606 & DQ268637 \\
\hline & CBS492.7 & USA-CO & DQ268604 & DQ268635 \\
\hline \multirow[t]{2}{*}{ O.ips } & ATCC-24285 & Canada & AY194937 & AY194949 \\
\hline & SYPT2 & Canada & AY194940 & AY194955 \\
\hline
\end{tabular}


Table 2. Cont.

\begin{tabular}{|c|c|c|c|c|}
\hline Species & Isolate & Location & ITS-LSU & $\beta$-tubulin \\
\hline \multirow[t]{16}{*}{ O. minus } & AU58.4 & Canada-BC & - & AY548743 \\
\hline & C348 & Canada & AY542496 & AY542507 \\
\hline & CFL873 & Canada & KC 305144 & KC336019 \\
\hline & DAOM-212686 & Canada & - & AY305690 \\
\hline & OM1 & UK & AY542494 & AY542505 \\
\hline & OM6 & USA-NE & AY542498 & AY542509 \\
\hline & CA-JM-81 & USA-CA & MZ297386 & MZ296749 \\
\hline & CA-JM-82 & USA-CA & MZ297387 & MZ296750 \\
\hline & CA-JM-83 & USA-CA & MZ297388 & MZ298931 \\
\hline & CA-JM-84 & USA-CA & MZ297389 & MZ296751 \\
\hline & CA-JM-85 & USA-CA & MZ297390 & MZ298932 \\
\hline & CA-JM-86 & USA-CA & MZ297391 & MZ296752 \\
\hline & CA-JM-87 & USA-CA & MZ297392 & MZ296753 \\
\hline & CA-JM-88 & USA-CA & MZ297393 & MZ296754 \\
\hline & CA-JM-89 & USA-CA & MZ297394 & MZ298933 \\
\hline & CA-JM-90 & USA-CA & MZ297395 & MZ296755 \\
\hline \multirow[t]{2}{*}{ O. montium } & ATCC-64697 & Canada & AY194941 & AY194957 \\
\hline & Kw430 & Canada & AY194948 & AY194964 \\
\hline \multirow[t]{4}{*}{ O. piceae } & B054 & Austria & HQ115733 & - \\
\hline & CNB-13 & Spain & AJ538341 & - \\
\hline & H2181 & Japan & AF211844 & AB934362 \\
\hline & KUC-2311 & Korea & - & DQ868379 \\
\hline \multirow[t]{2}{*}{ O. piliferum } & 201/1a & United Kingdom & - & AF221629 \\
\hline & CBS129.32 & Netherlands & - & AY305705 \\
\hline \multirow[t]{2}{*}{ O. pseudotsugae } & $92-634 / 302 / 6$ & Canada-BC & AY542502 & AY542510 \\
\hline & OM5 & USA-ID & AY542500 & AY548744 \\
\hline \multirow[t]{2}{*}{ O. setosum } & AU160-27 & Canada & - & AY789160 \\
\hline & NZFS-3652-1 & New Zealand & - & AY789159 \\
\hline \multirow[t]{2}{*}{ O. subalpinum } & YCC-408 & Japan & - & AB200429 \\
\hline & YCC-410 & Japan & - & AB200430 \\
\hline
\end{tabular}

\subsection{Prevalence of Fungi in Beetle Maxillae and Pellet, and in Mites}

We analyzed the prevalence of two fungal species in two different locations on the beetle, by fitting a generalized linear mixed model (GLMM) with a binomial response and logit link using the fixed effects of fungal species and location, as well as their interaction. Since each beetle had four observations, we included a random beetle effect in the model. Similarly, we fitted a GLMM model to the mite data using species as a fixed effect and beetle ID as a random effect. We performed these analyses in R version 4.0.5 [38].

\subsection{Fungal Growth Rates across a Temperature Gradient and Interactions with Yeast}

We grew eight (O. minus) and nine (G. clavigera) pure strain subsamples of the characterized blue-stain fungi in incubators (Thermo Scientific ${ }^{\mathrm{TM}}$ Precision 818, Marietta, $\mathrm{OH}$, USA) at $5{ }^{\circ} \mathrm{C}$ intervals from -10 to $30{ }^{\circ} \mathrm{C}$ and at $2{ }^{\circ} \mathrm{C}$ intervals from 32 to $36^{\circ} \mathrm{C}$. We crossmarked the MEA plates at $90^{\circ} \mathrm{C}$ and averaged two perpendicular diameter measurements to estimate the growth of every single strain of blue-stain fungi and of a replicate that had been allowed to grow for 7-days with the most prevalent yeast species. Physiological characters measured included the temperature at which minimum, maximum, and optimal growth rates were achieved by each blue-stain fungus, and in combination with the yeast. We used a Gaussian GLMM with a random effect for strain to fit the growth measurements to the predictors of fungal species, categorical temperature increment, whether yeast was present, and an interaction between all three variables. 


\section{Results}

\subsection{Phylogenetic Analyses}

The combined Ophiostoma ITS2-LSU + Btubulin dataset consisted of 34 ingroup sequences with a total of 1566 positions (961 for ITS2-LSU and 605 for $\beta$-tubulin) while the Grosmannia ITS2-LSU + Btubulin dataset consisted of 32 ingroup sequences with a total of 1380 positions (871 for ITS2-LSU and 509 for $\beta$-tubulin). In the analyses of the Ophiostoma dataset, JPB isolates clustered in two clades: one consisting of three JPB isolates and O. minus isolates from Canada and the United States, and another consisted only of JPB isolates (Figure 2). When we compared $\beta$-tubulin sequences within the isolates of the first clade, we found that they differed in 4-6 nucleotides, whereas when we compared isolates between the two clades, they differed in about 17 nucleotides. Thus, isolates from the first clade are closely related to O. minus, while the other clade may not represent O. minus. In addition, isolate OM1 from the UK differed from isolate OM6 in more than 60 nucleotides. This suggested that this isolate may not have represented O. minus. When we compared the ITS2-LSU sequences, we found only three differences between these two clades. However, we were able to compare only portions of the ITS2 region since there were not complete ITS2-LSU sequences for O. minus available in GenBank. In the analyses of the Grosmannia dataset (Figure 3), all the isolates obtained from JPB clustered within the Grosmannia clavigera clade. The Grosmannia clavigera clade consisted of two groups, but these isolates differed only in one nucleotide in the ITS2-LSU dataset with no differences in the $\beta$-tubulin dataset. This confirmed that these JPB isolates likely represent $G$. clavigera.

The most prevalent microorganism we recovered from JPBs maxillae, pellet, and mites was a yeast (Figure 4). Based on cultural characters of the yeast we separated from uncommon species present in the sample. From these, we sequenced strain CA-JM-92 obtaining only the ITS sequence which was compared with other species using BLAST Search tool (the nearest match for this isolate was the strain CBS:9990, KY103918, with a 99\% Percent Identity and Query Cover of 97\%). Using molecular data, we identified the yeast as Kuraishia molischiana Dlauchy, G. Péter, Tornai-Leh. \& Kurtzman [39]. This species was recently split from K. capsulata, which was the only Kuraishia spp. previously reported from JPB. We also recovered two blue-stain fungi frequently, these represented an Ophiostoma and a Leptographium (a common anamorph of Grosmannia) species. We did not find any other previously documented JPB blue-stain fungal associates. Morphological and molecular identification (Figure 2) indicated that the Ophiostoma species represented $O$. minus. However, the Leptographium did not agree morphologically with all characters of G. clavigera as none of the strains produced the long, clavate, and septate conidia characteristic of G. clavigera, but molecularly matched that species closely (Figure 3). Although we found a high frequency of $G$. clavigera in our sample, we found the antagonistic O. minus in moderate frequency. The latter species is an indicator of collapsing southern pine beetle populations in southeastern USA $[4,40]$.

\subsection{Prevalence of Fungal Associates on Beetles and Mites}

Grosmannia clavigera was most prevalent in the maxillae (probability of presence $=0.79$ ), and less prevalent in the pellet (probability of presence $=0.34$ ) and this difference was significant (Table 3). However, O. minus had a similar probability of presence in each location (Table 3).

The presence of both fungi, as well as the yeast in fecal pellets, indicated their capacity of surviving digestion; however, their prevalence in these samples varied. The yeast K. molischiana was present in both beetle structures and only slightly less on mites which suggested its capacity to exploit many dispersal strategies. Similarly, O. minus rate of recovery from the pellet was not different from its occurrence on the JPB maxillae (Table 3, Figure 5). In contrast, G. clavigera was significantly less likely to show up in the fecal pellets than in the maxillae (Table 3). Overall, there was a low frequency of blue-stain fungi on mites (Figure 4). However, we found mites to carry O. minus more frequently than 
G. clavigera $\left(\chi^{2}=7.07, p \leq 0.01, n=17\right)$, and overall, within the system $O$. minus was more abundant on mites.

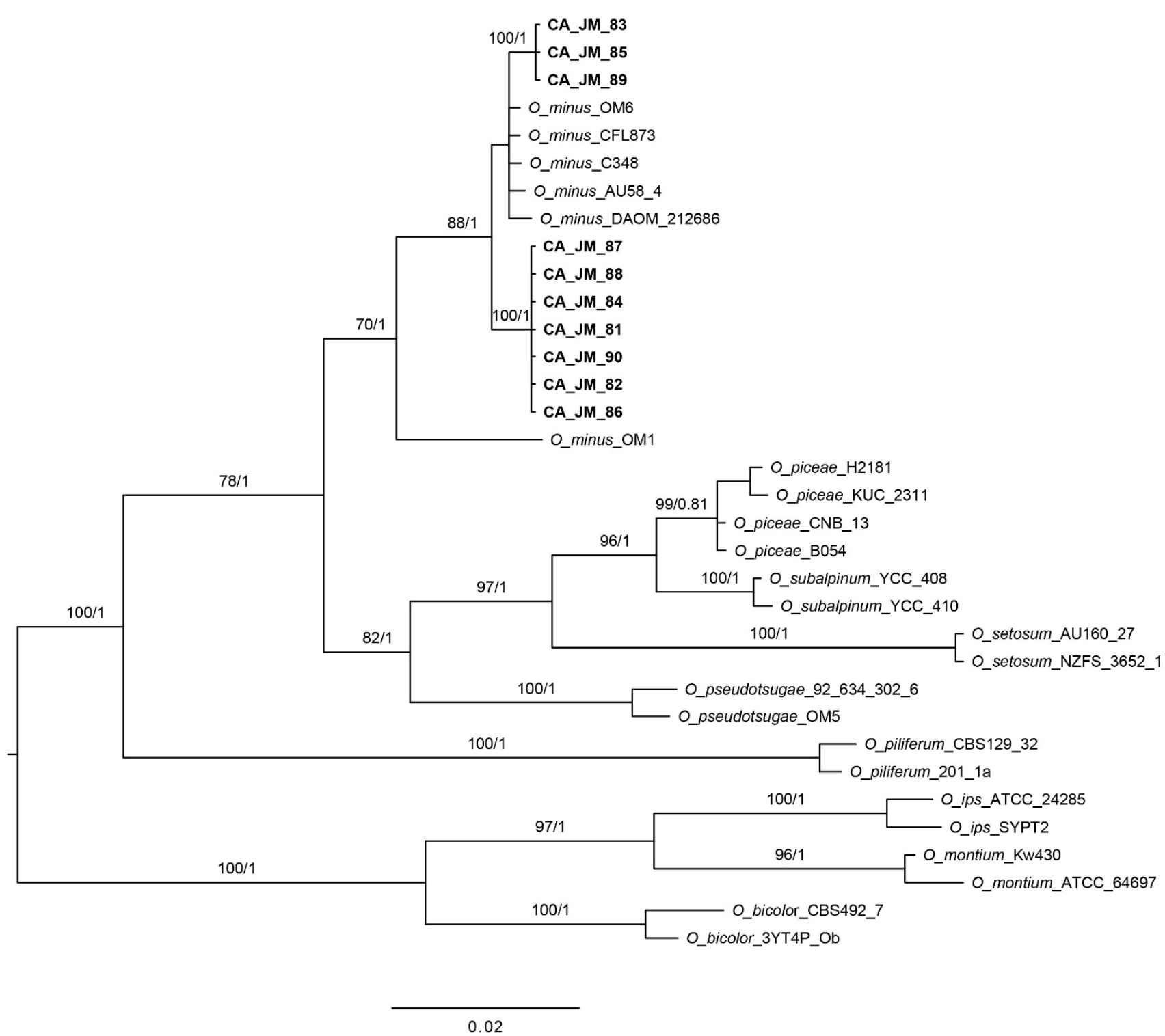

Figure 2. Phylogeny of Ophiostoma species as inferred from BI analyses of ITS2-LSU and B-tubulin sequences. Support values along branches are from ML Bootstrap $(\geq 50 \%)$ and Bayesian posterior probabilities ( $\mathrm{PP} \geq 0.80$ ), respectively.

\subsection{Yeast Interaction with Blue-Stain Fungal Growth across a Temperature Gradient}

In general, O. minus grew well over a wider range of temperatures than G. clavigera, especially in temperatures above $20^{\circ} \mathrm{C}$. In fact, the optimal growth temperature of G. clavigera was lower at $19.24{ }^{\circ} \mathrm{C}$ than the $25.06{ }^{\circ} \mathrm{C}$ of $\mathrm{O}$. minus and that did not change when growing in association with the yeast $\mathrm{K}$. molischiana, when these were $19.05^{\circ} \mathrm{C}$ and $24.90^{\circ} \mathrm{C}$, respectively. We found that when not paired with the yeast, the growth of the two blue-stain fungi was nearest from 5 to $20^{\circ} \mathrm{C}$ (see significant differences in Figure 6A), but $O$. minus maintained a growth advantage from 5 to $15^{\circ} \mathrm{C}$ (Figure $6 \mathrm{~A}$ ). However, an inverse growth advantage, benefiting G. clavigera over O. minus, was evident in that same temperature range (not significant at $5^{\circ} \mathrm{C}$ ) when each blue-stain grew in association with the yeast (Figure 6B). However, above $20^{\circ} \mathrm{C}$, there was a small advantage in the growth of O. minus contrasting to when it grew alone (Figure $6 \mathrm{~B}$ ). 


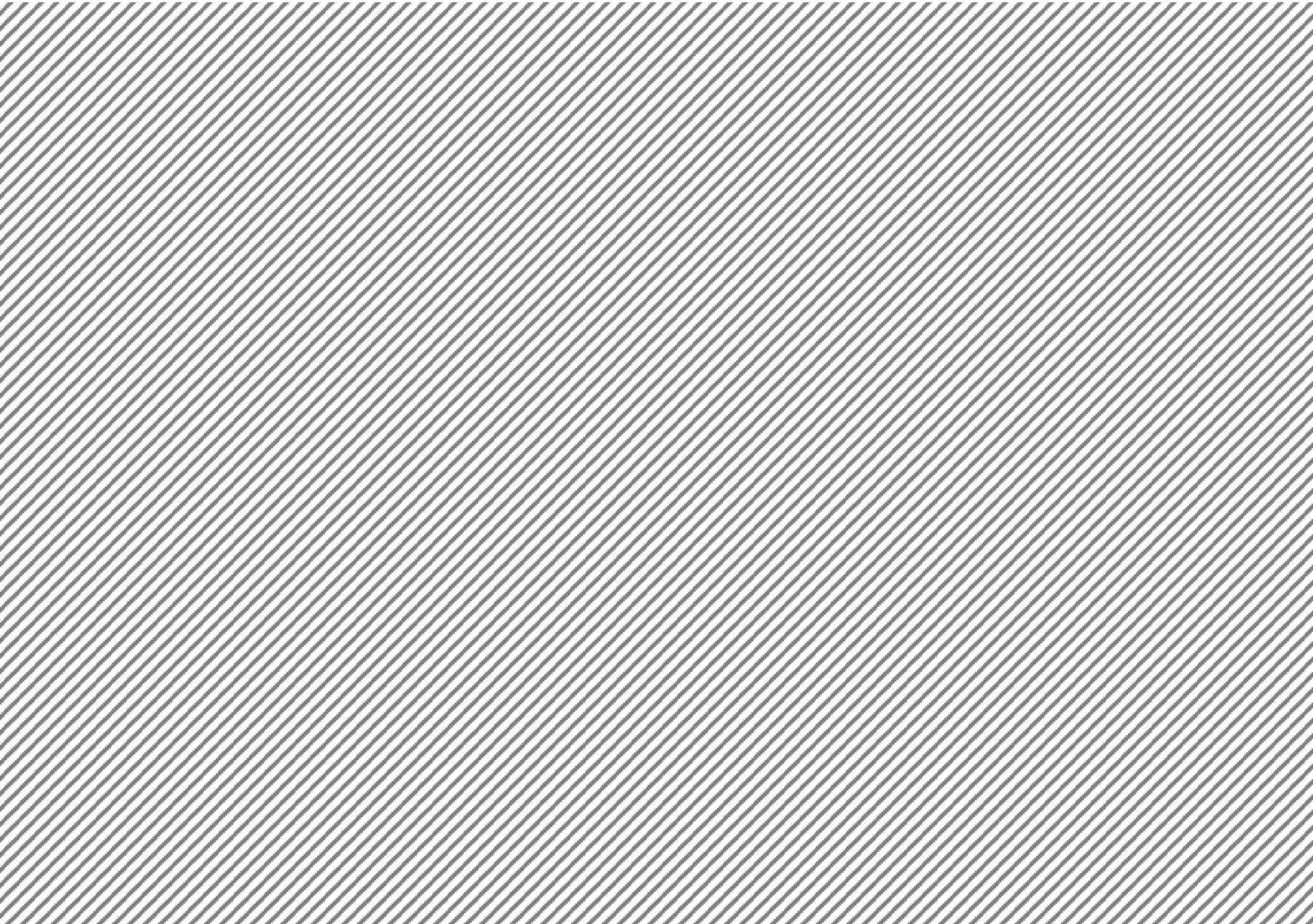

0.004

Figure 3. Phylogeny of the Grosmannia clade as inferred from BI analyses of ITS2-LSU and B-tubulin sequences. Support values along branches are from ML Bootstrap $(\geq 50 \%)$ and Bayesian posterior probabilities (PP $\geq 0.80)$, respectively.

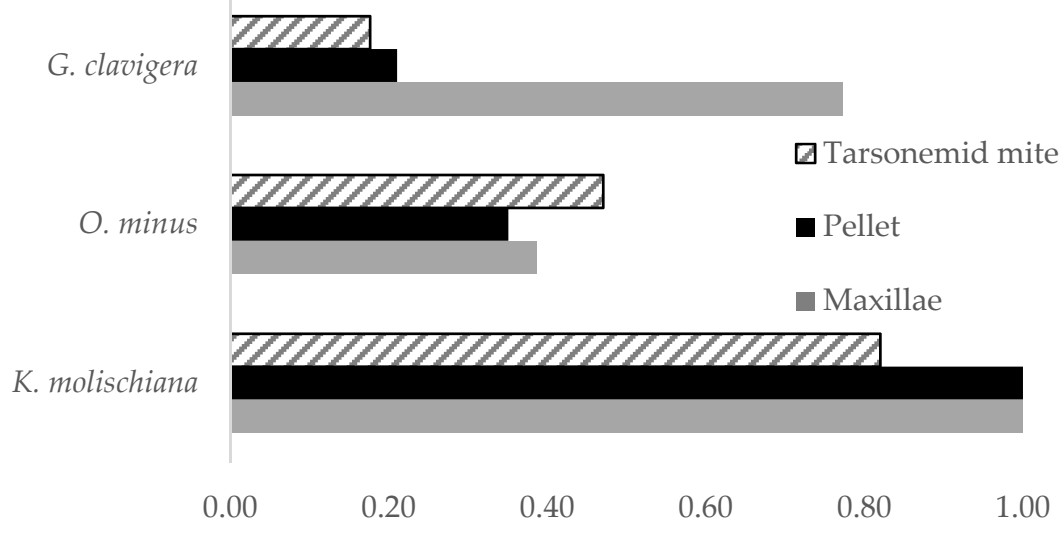

Figure 4. Percent frequency of three Jeffrey pine beetle fungal associates in tarsonemid mites, pellet, maxillae, from an endemic-population sample. 
Table 3. Grosmannia clavigera was significantly more likely to be present in Jeffrey pine beetle's maxillae than in its fecal pellet, while Ophiostoma minus was equally likely to be found on either.

\begin{tabular}{ccccc}
\hline Fungal Species & Value & DF & Chisq & Pr (>Chisq) \\
\hline Maxillae-Pellet: G. clavigera & 0.95 & 1 & 28.87 & $<0.001$ \\
Maxillae-Pellet: O. minus & 0.52 & 1 & 0.04 & 0.833 \\
\hline
\end{tabular}
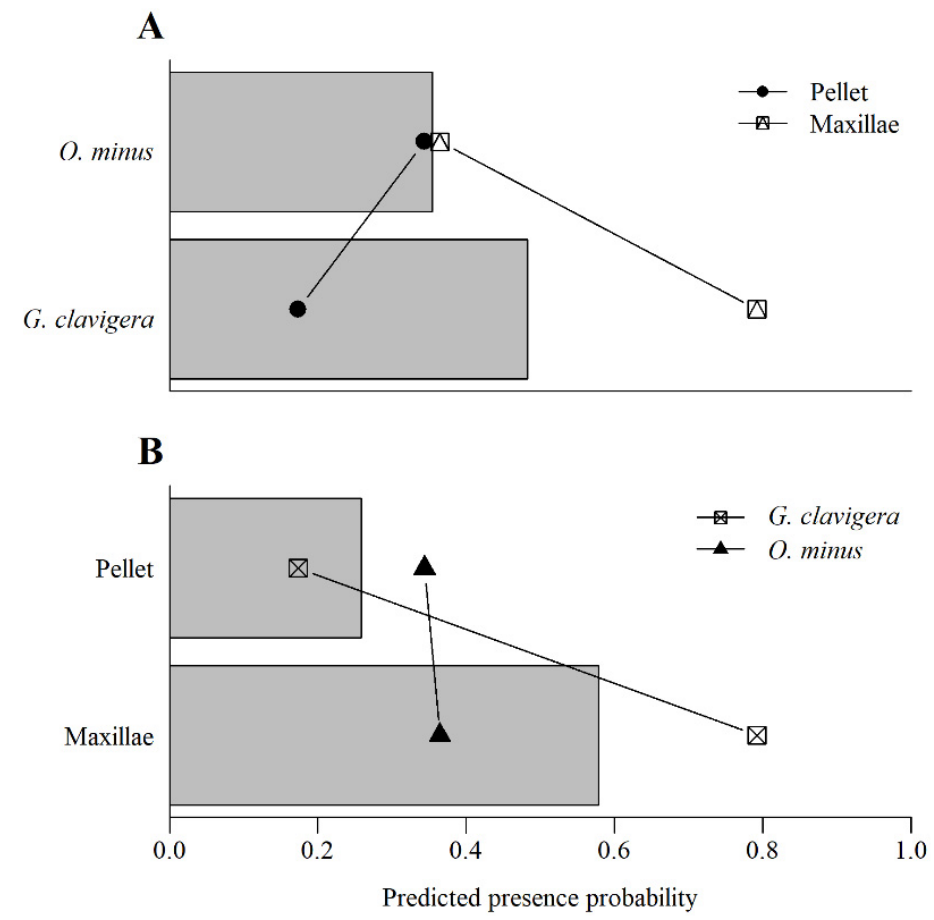

Figure 5. Predicted probability of presence of fungal associates O. minus and G. clavigera with the location interaction (A) and predicted probability of presence within by location with the fungal associate interaction (B). Main effects (averaged across species or location) are shown by bars while interaction effects, which allowed each fungal species to differ in presence between locations, are shown by points and lines.

A

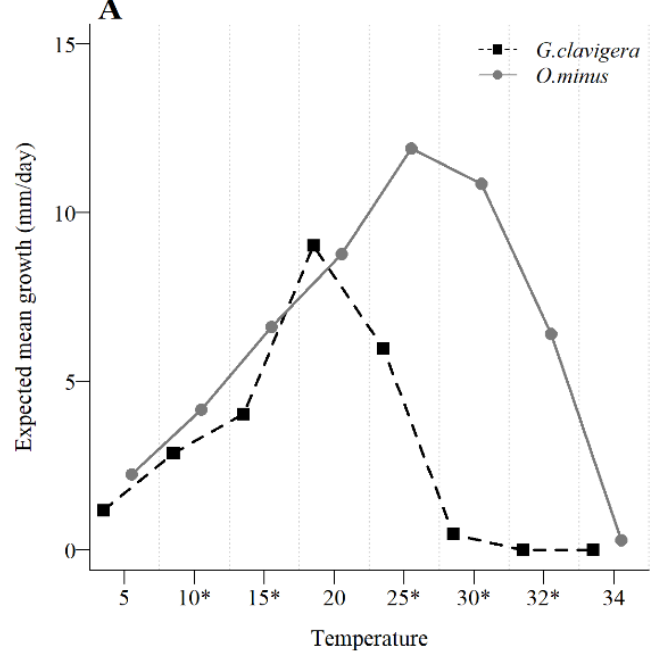

B

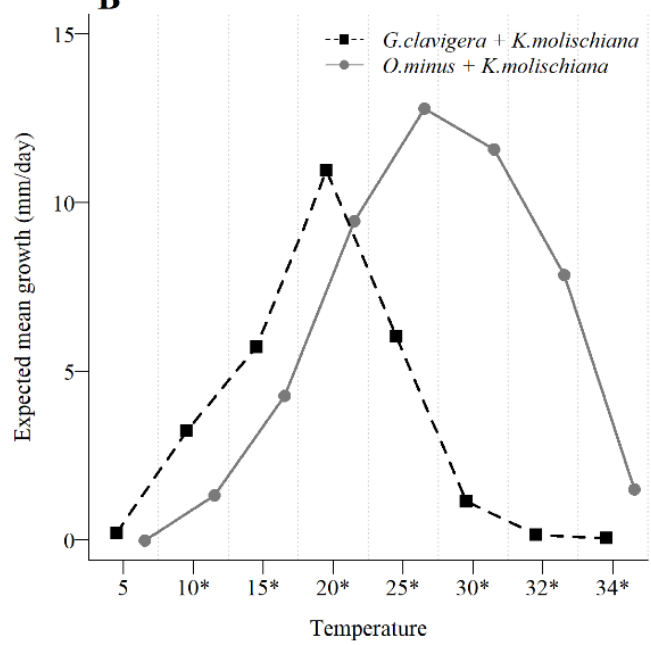

Figure 6. The expected average growth of nine isolates of the fungi Grosmannia clavigera and eight of Ophiostoma minus (A) and after their paired growth with the yeast Kuraishia molischiana (B), showed that while growing alone O. minus generally had a growth advantage over G. clavigera that reversed from 5 to $15^{\circ} \mathrm{C}$ when both fungi grew in combination with the yeast. Significant differences are indicated with an asterisk $\left(^{*}\right)$. 


\section{Discussion}

Examining symbiotic associations of bark beetles from populations that are in equilibrium (endemic-level) or decreasing, often requires considerably more effort than studying irruptive ones, but only by studying endemic populations can we better understand the factors constraining them. Among Dendroctonus bark beetles, the Jeffrey pine beetle is the closest morphologically, genetically, and ecologically to the MPB, thus factors affecting its ecology may be similar to those in the more ecologically impactful MPB, making our findings relevant to understanding the MPB. For the first time, here we describe the fungal associates of an endemic JPB population. Although small in comparison, our study results contrast with a previous study that examined the fungal fauna of JPB across its distribution from a non-endemic population [17] by detecting the presence of an antagonistic fungus associated with its symbiotic system. The population level in the previous study was moderately high based on reports [22], as well as from personal comments made by Diana Six (17 February 2018), in contrast to our studied population.

As also found by Six and Paine [17], we found G. clavigera to be the most prevalent blue-stain fungal associate carried in the beetles' maxillae in our endemic population sample. This is important since in the MPB symbiosis, G. clavigera is required (obligate mutualist) by beetles to reach their adult phase [41] and its function in the JPB symbiotic system is either similar or even more important, given the phylogenetic proximity of both insects and their fungi, and especially since this is the only mutualistic blue-stain fungus in that symbiosis. It is also important since a reduction of this and other MPB mutualists might be associated with a reduction in population size leading to endemic population levels [5].

Although showing molecular affinity to G. clavigera, strains obtained from JPB differed morphologically in that their conidia were never typical for the species, but similar observations were reported previously [17]. To verify if the growth media was affecting our observations, we cultured G. clavigera from MPB voucher isolates in our MEA media and contrary to our observation from JPB isolates obtained vigorous production of its typical conidia. We saw the same lack of typical conidia production when we cultured G. clavigera in ponderosa pine phloem; however, this has a different oleoresin composition from Jeffrey pine that could have affected our observations. Moreover, Paine and Hanlon [42] found that $\alpha$-pinene, a primary component in the oleoresin of ponderosa pine reduced the growth of $G$. clavigera isolates from JPB while $n$-heptane, the principal oleoresin component of Jeffrey pine enhanced its growth. Therefore, it is probable that, as in their beetle carriers, G. clavigera in these two bark beetles may be differentiating into distinct species and further studies contrasting specimens from the two beetles may show that to be the case.

Finding O. minus was surprising. This fungus is antagonistic to the southern pine beetle, causing their populations to collapse when the beetle's phoretic tarsonemid mites transporting the fungus increase in number [4]. This new record of blue-stain fungi from JPB may have resulted from the lateral transmission from mites carried to the attacked tree by Ips emarginatus LeConte, as tarsonemid mites and O. minus are carried by that species [18]. This bark beetle was present in the tree from which our collection was made but not sampled in our study (Danny Cluck, personal comment). Tarsonemid mites in our sample were scarce and many did not carry fungi, but mites carried O. minus more often than G. clavigera (Figure 4). Therefore, it is probable that the recruitment of O. minus by JPB is facilitated by phoretic mites moving from I. emarginatus to the beetle. Moreover, once within the phloem, O. minus is ingested by JPB and can become dispersed within the beetle's niche making it more prevalent, reducing the reproductive success of the beetle. Confirming the lateral transmission of O. minus carried by I. emarginatus phoretic tarsonemid mites into the niche of the JPB could have important management implications. For example, we could attempt to reduce the reproductive success of JPB by influencing I. emarginatus to attack trees under the attack of JPB. Additionally, the presence of I. emarginatus in trees attacked by JPB may help us predict future shifts in JPB populations. A question that emerges from these findings is, what mechanisms influence 
the prevalence or reduction of $O$. minus from JPB populations? Future research should include analyzing fungal associates in different JPB population levels as well as include the sampling of I. emarginatus fungal associates and should relate findings to weather factors such as temperature and the ecosystems relative humidity which drive variations in fungal diversity. This will strengthen the hypothesis that antagonistic fungi act as endogenous bark beetle population regulators and what may drive their prevalence.

The topic of bark beetle fungal associates' survival from digestion remains largely unknown, especially when looking at it as a means for their dissemination. Rivera and others [9] described the occurrence of yeasts in specific gut areas in several Dendroctonus species. They were able to grow species found in other Dendroctonus species hindguts, but their efforts were not aimed at detecting yeast dissemination strategies. The ubiquity of the yeast K. molischiana in both mycangia and pellet, as well as in the mites, suggests that this yeast does not have a dependency on beetles or mites for its dispersion and that it can exploit elimination. Dissemination of blue-stain fungi is also poorly understood. Dissemination of $O$. minus by JPB is secondary to its mite dissemination and its high survival rate in pellets, inferring that ingestion is similar to its prevalence in the mycangia suggest dissemination in beetle's elimination is exploited by this fungus. Its unspecialized dissemination strategy suggests that as K. molischiana, it has a lack of dependence on JPB for its survival. In contrast to these two species, the higher frequency of G. clavigera in maxillary mycangia combined with its higher reduction during beetle elimination reflects the strong association of this species to JPB. These findings suggest that understanding more about the dissemination strategies of bark beetle associates may be useful to understand their role in their carrier beetle's symbiosis but that complex interactions require further experimentation to be discerned. As hypothesized, a mutualistic fungus G. clavigera was more prevalent in JPB especially in what is considered a highly derived structure for mutualists dissemination, the mycangia, while a different fungus, in this case, a known Dendroctonus antagonist was more prevalent in mites.

Given the limitations of our study, the way in which K. molischiana affects the performance of G. clavigera deserves to be investigated in further detail. Yeasts such as K. molischiana cover the growth of filamentous fungi completely in culture, making their isolation difficult. Although we did not study the insulating effects of yeast coating in fungi at cold temperatures, it is possible that its compounds may provide such protection to G. clavigera. However, this does not entirely explain why a similar effect is not seen in O. minus. Thus, the thermal requirements of this warm temperature-loving species may not be met by the potential insulation provided by the yeast. However, this does not mean that the interaction of $O$. minus with the yeast is entirely antagonistic and further studies should focus on defining their relationship. Nevertheless, a mutualistic interaction was evident between G. clavigera and K. molischiana that may improve tree host establishment in priority of other fungal species, in this case, O. minus. The interaction is also indirectly mutualistic with JPB as it improves the incidence of its mutualist fungi over an antagonist. Our findings set a start point for further studies that will continue elucidating the complexity of these and similar interactions between insect microbial associates.

Author Contributions: Conceptualization, J.E.M.; methodology, J.E.M. and B.O.-S.; software, S.L.K. and B.O.-S.; formal analysis, S.L.K. and B.O.-S.; investigation, J.E.M.; data curation, J.E.M.; writingoriginal draft preparation, J.E.M.; writing-review and editing, B.O.-S. and S.L.K. All authors have read and agreed to the published version of the manuscript.

Funding: This research received no external funding.

Institutional Review Board Statement: Not applicable.

Informed Consent Statement: Not applicable.

Data Availability Statement: The datasets generated during and/or analyzed during the current study were submitted to TreeBase. Sequence data that support the findings of this study have 
been deposited in GenBank. All data generated or analyzed during this study are included in this published article. All specimens described in this study were deposited in institutional herbaria.

Acknowledgments: This work would not have been possible without the help of Forest Health Products entomologists Danny Cluck (Pacific Southwest Region) who provided us with the JPB samples and the excellent and arduous work by technicians Danielle Francis and Eddyliz P. Rosado. Danielle went beyond her duties providing ideas to improve the essays. We also thank the help of two anonymous reviewers. The USDA/FS RMRS and NRS Research Stations provided support to the authors during their research. The Hispanic Association of Colleges and Universities (HACU) provided support for our excellent technicians.

Conflicts of Interest: The authors declare no conflict of interest. The funders had no role in the design of the study; in the collection, analyses, or interpretation of data; in the writing of the manuscript, or in the decision to publish the results.

\section{References}

1. Wang, Y.; Lim, L.; Madilao, L.; Lah, L.; Bohlmann, J.; Breuil, C. Gene discovery for enzymes involved in limonene modification or utilization by the mountain pine beetle-associated pathogen Grosmannia clavigera. Appl. Environ. Microbiol. 2014, 80, 4566-4576. [CrossRef]

2. Hunt, D.W.A.; Borden, J.H. Conversion of verbenols to verbenone by yeasts isolated from Dendroctonus ponderosae (Coleoptera: Scolytidae). J. Chem. Ecol. 1990, 16, 1385-1397. [CrossRef]

3. Barras, S.J. Antagonism between Dendroctonus frontalis and the fungus Ceratocystis minor. Ann. Entomol. Soc. Am. 1970, 63, 1187-1190. [CrossRef]

4. Hofstetter, R.W.; Cronin, J.T.; Klepzig, K.D.; Moser, J.C.; Ayres, M.P. Antagonisms, mutualisms and commensalisms affect outbreak dynamics of the southern pine beetle. Oecologia 2006, 147, 679-691. [CrossRef]

5. Mercado, J.E.; Ortiz-Santana, B.; Kay, S.L. Fungal Frequency and Mite Load Trends Interact with a Declining Mountain Pine Beetle Population. Forests 2018, 9, 484. [CrossRef]

6. Bridges, J.R.; Moser, J.C. Role of two phoretic mites in transmission of bluestain fungus, Ceratocystis minor. Ecol. Entomol. 1983, 8 , 9-12. [CrossRef]

7. Mercado, J.E.; Hofstetter, R.W.; Reboletti, D.M.; Negrón, J.F. Phoretic symbionts of the mountain pine beetle (Dendroctonus ponderosae Hopkins). For. Sci. 2014, 60, 512-526. [CrossRef]

8. Rivera, F.N.; González, E.; Gómez, Z.; López, N.; Hernández-Rodríguez, C.; Berkov, A.; Zuñiga, G. Gut-associated yeast in bark beetles of the genus Dendroctonus Erichson (Coleoptera: Curculionidae: Scolytinae). Biol. J. Linn. Soc. 2009, 98, 325-342. [CrossRef]

9. Davis, T.S. The ecology of yeasts in the bark beetle holobiont: A century of research revisited. Microb. Ecol. 2015, 69, 723-732. [CrossRef] [PubMed]

10. Adams, A.S.; Six, D.L. Temporal variation in mycophagy and prevalence of fungi associated with developmental stages of Dendroctonus ponderosae (Coleoptera: Curculionidae). Environ. Entomol. 2014, 36, 64-72. [CrossRef]

11. Farmer, L.J. The Phloem-Yeast Complex during Infestations of the Mountain Pine Beetle in Lodgepole Pine. Ph.D. Thesis, University of Utah, Salt Lake City, UT, USA, 1965.

12. Wood, S.L. The Bark and Ambrosia Beetles of North and Central America (Coleoptera: Scolytidae), a Taxonomic Monograph; Great Basin Nat. (Mem. 6); Brigham John University: Provo, UT, USA, 1982; pp. 150-203.

13. Kelley, S.T.; Farrell, B.D. Is specialization a dead end? The phylogeny of host use in Dendroctonus bark beetles (Scolytidae). Evolution 1998, 52, 1731-1743. [CrossRef]

14. Reeve, J.D.; Anderson, F.E.; Kelley, S.T. Ancestral state reconstruction for Dendroctonus bark beetles: Evolution of a tree killer. Environ. Entomol. 2012, 41, 723-730. [CrossRef] [PubMed]

15. Victor, J.; Zuñiga, G. Phylogeny of Dendroctonus bark beetles (Coleoptera: Curculionidae: Scolytinae) inferred from morphological and molecular data. Syst. Entomol. 2016, 41, 162-177. [CrossRef]

16. Whitney, H.S.; Farris, S.H. Maxillary mycangium in the mountain pine beetle. Science 1970, 167, 54-55. [CrossRef]

17. Six, D.L.; Paine, T.D. Ophiostoma clavigerum is the mycangial fungus of the Jeffrey pine beetle, Dendroctonus jeffreyi. Mycologia 1997, 89, 858-866. [CrossRef]

18. Mathre, D.E. Survey of Ceratocystis spp. associated with bark beetles in California. Contrib. Boyce Thompson Inst. 1964, 22, 353-362. [CrossRef]

19. Alamouti, S.M.; Wang, V.; Diguistini, S.; Six, D.L.; Bohlmann, J.; Hamelin, R.C.; Feau, N.; Breuil, C. Gene genealogies reveal cryptic species and host preferences for the pine fungal pathogen Grosmannia clavigera. Mol. Ecol. 2011, 20, 2581-2602. [CrossRef]

20. Shifrine, M.; Phaff, H.J. The association of yeasts with certain bark beetles. Mycologia 1956, 48, 41-55. [CrossRef]

21. Bleiker, K.P.; Potter, S.E.; Lauzon, C.R.; Six, D.L. Transport of fungal symbionts by mountain pine beetles. Can. Entomol. 2009, 141, 503-514. [CrossRef]

22. California Forest Pest Council. Available online: https://caforestpestcouncil.org/tag/pest-condition-reports / (accessed on 9 January 2020). 
23. Solheim, H.; Krokene, P. Growth and virulence of mountain pine beetle associated blue-stain fungi, Ophiostoma clavigerum and Ophiostoma montium. Can. J. Bot. 1998, 76, 561-566. [CrossRef]

24. Upadhyay, H.P. A Monograph of Ceratocystis and Ceratocystiopsis; University of Georgia Press: Athens, GA, USA, 1981; 176p.

25. Lee, S.; Kim, J.J.; Breuil, C. Leptographium longiclavatum sp. nov., a new species associated with the mountain pine beetle, Dendroctonus ponderosae. Mycol. Res. 2005, 109, 1162-1170. [CrossRef] [PubMed]

26. Benson, D.A.; Cavanaugh, M.; Clark, K.; Karsch-Mizrachi, I.; Lipman, D.J.; Ostell, J.; Sayers, E.W. GenBank. Nucleic Acids Res. 2013, 41, D36-D42. [CrossRef]

27. Kim, J.-J.; Kim, S.H.; Lee, S.; Breuil, C. Distinguishing Ophiostoma ips and Ophiostoma montium, two bark beetle-associated sapstain fungi. FEMS Microbiol. Lett. 2003, 222, 187-192. [CrossRef]

28. Gorton, C.; Kim, S.H.; Henricot, B.; Webber, J.; Breuil, C. Phylogenetic analysis of the bluestain fungus Ophiostoma minus based on partial ITS rDNA and beta-tubulin gene sequences. Mycol. Res. 2004, 108, 759-765. [CrossRef]

29. Zipfel, R.D.; De Beer, Z.W.; Jacobs, K.; Wingfield, B.D.; Winfield, M.J. Multi-gene phylogenies define Ceratocystiopsis and Grosmannia distinct from Ophiostoma. Stud. Mycol. 2006, 55, 75-97. [CrossRef]

30. Roe, A.D.; Rice, A.V.; Bromilow, S.E.; Cooke, J.E.K.; Sperling, F.A.H. Multilocus species identification and fungal DNA barcoding: Insights from blue stain fungal symbionts of the mountain pine beetle. Mol. Ecol. Resour. 2010, 10, 946-959. [CrossRef]

31. Six, D.L.; De Beer, Z.W.; Duong, T.A.; Carroll, A.L.; Wingfield, M.J. Fungal associates of the lodgepole pine beetle, Dendroctonus murrayanae. Antonie Van Leuwenhoek 2011, 100, 231-244. [CrossRef]

32. Miller, M.A.; Pfeiffer, W.; Schwartz, T. Creating the CIP-RES Science Gateway for inference of large phylogenetic trees. In Proceedings of the Gateway Computing Environments Workshop (eds. GCE), New Orleans, LA, USA, 14 November 2010; pp. 1-8.

33. Larsson, A. AliView: A fast and lightweight alignment viewer and editor for large datasets. Bioinformatics 2014, 30, 3276-3278. [CrossRef]

34. Gouy, M.; Guindon, S.; Gascuel, O. SeaView Version 4: A multiplatform graphical user interface for sequence alignment and phylogenetic tree building. Mol. Biol. Evol. 2010, 27, 221-224. [CrossRef]

35. Darriba, D.; Taboada, G.L.; Doallo, R.; Posada, D. jModelTest 2: More models, new heuristics and parallel computing. Nat. Methods 2012, 9, 772. [CrossRef] [PubMed]

36. Ronquist, F.; Teslenko, M.; Van Der Mark, P.; Ayres, D.L.; Darling, A.; Höhna, S.; Larget, B.; Liu, L.; Suchard, M.A.; Huelsenbeck, J.P. MrBayes 3.2: Efficient Bayesian phylogenetic inference and model choice across a large model space. Syst. Biol. 2012, 61, 539-542. [CrossRef]

37. Rambaut, A. FigTree v1.4.4. Institute of Evolutionary Biology, University of Edinburgh. Available online: http://tree.bio.ed.ac. uk/software/figtree (accessed on 11 March 2021).

38. R: A Language and Environment for Statistical Computing. R Foundation for Statistical Computing, Vienna, Austria. Available online: https: / / www.R-project.org/ (accessed on 9 March 2020).

39. Péter, G.; Dlauchy, D.; Tornai-Lehoczki, J.; Kurtzman, C.P. Kuraishia molischiana sp. nov., the teleomorph of Candida molischiana. Antonie van Leeuwenhoek 2005, 88, 241-247. [CrossRef] [PubMed]

40. Lombardero, M.J.; Ayres, M.P.; Hofstetter, R.W.; Moser, J.C.; Lepzig, K.D. Strong indirect interactions of Tarsonemus mites (Acarina: Tarsonemidae) and Dendroctonus frontalis (Coleoptera: Scolytidae). Oikos 2003, 102, 243-252. [CrossRef]

41. Myrholm, C.L.; Langor, D.W. Assessment of the impact of symbiont Ophiostomatales (Fungi) on mountain pine beetle (Coleoptera: Curculionidae) performance on a jack pine (Pinaceae) diet using a novel in vitro rearing method. Can. Entomol. 2016, 148, 68-82. [CrossRef]

42. Paine, T.D.; Hanlon, C.C. Influence of oleoresin constituents from Pinus ponderosa and Pinus jeffreyi on growth of mycangial fungi from Dendroctonus ponderosae and Dendroctonus jeffreyi. J. Chem. Ecol. 1994, 20, 2551-2563. [CrossRef] [PubMed] 